\title{
Charge Ordering in Half-Doped Manganites: Weak Charge Dis- proportion and Leading Mechanisms
}

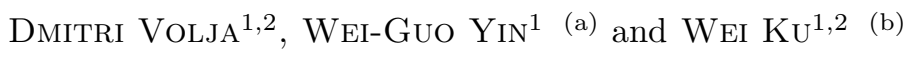 \\ 1 Condensed Matter Physics and Materials Science Department, Brookhaven National Laboratory, Upton, New York \\ 11973, USA \\ 2 Physics Department, State University of New York, Stony Brook, New York 11790, USA
}

PACS $75.47 . \mathrm{Lx}$ - Manganites (magnetotransport materials)
PACS $71.45 . \mathrm{Lr}$ - Charge-density waves - collective excitations
PACS $71.10 . \mathrm{Fd}$ - Lattice fermion models (Hubbard model, etc.)
PACS $71.30 .+\mathrm{h}$ - Metal-insulator transitions and other electronic transitions

\begin{abstract}
The apparent contradiction between the recently observed weak charge disproportion and the traditional $\mathrm{Mn}^{3+} / \mathrm{Mn}^{4+}$ picture of the charge-orbital orders in half-doped manganites is resolved by a novel Wannier states analysis of the $\mathrm{LDA}+U$ electronic structure. Strong electron itinerancy in this charge-transfer system significantly delocalizes the occupied low-energy " $\mathrm{Mn}^{3+}$ " Wannier states such that charge leaks into the " $\mathrm{Mn}^{4+}$ "-sites. Furthermore, the leading mechanisms of the charge order are quantified via our first-principles derivation of the low-energy effective Hamiltonian. The electron-electron interaction is found to play a role as important as the electronlattice interaction.
\end{abstract}

Introduction. - The exploration of interplay among distinct orders lies in the heart of condensed matter physics and materials science, as this interplay often give rises to tunable properties of practical applications, such ás exotic states and colossal responses to external stimuli. Manganese oxides such as $\mathrm{La}_{1-x} \mathrm{Ca}_{x} \mathrm{MnO}_{3}$, which host rich charge, orbital, spin, and lattice degrees of freedom, have thus attracted great attention [1]. In particular, the vastly interesting colossal magnetoresistance (CMR) effect for $x \sim 0.2-0.4$ exemplifies rich physics originating from proximity of competing orders. In a slightly more doped system $(x=0.5)$, all these orders coexist in an insulating state [2, providing a unique opportunity for a clean investigation of the strength and origin of each order. Therefore, the study of half-doped manganites is key to a realistic understanding of the physics of manganites in general and the CMR effect in particular [3,4].

The peculiar multiple orders in half-doped manganites have long been understood in the Goodenough model [2] of a $\mathrm{Mn} 3+/ 4+$ checkerboard charge order $(\mathrm{CO})$ with the occupied $\mathrm{Mn}^{3+} e_{g}$ orbitals zigzag ordered in the CE-type antiferromagnetic background [2]. Pertaining to CMR, it is broadly believed that a key is the emerging of nanoscale

\footnotetext{
(a) E-mail: wyin@bnl.gov

(b) E-mail: weiku@bnl.gov
}

charge-ordered insulating regions of Goodenough type at intermediate temperatures, which could melt rapidly in the magnetic field [4,5].

Nonetheless, the simple yet profound Goodenough picture has been vigorously challenged thanks to recent experimental observations of nearly indiscernible charge disproportion (CD) in a number of half-doped manganites 6 13. Such weak CD has also been observed in firstprinciples computations [14, 15] and charge transfer between $\mathrm{Mn}$ and $\mathrm{O}$ sites was reported as well [15]. In essence, these findings have revived a broader discussion on the substantial mismatch of valence and charge in most charge-transfer insulators. Indeed, extensive experimental and theoretical effort has been made in light of the novel Zener-polaron model 9,33 in which all the Mn sites become equivalent with valence being +3.5. Amazingly, most of these investigations concluded in favor of two distinct Mn sites as predicted in the Goodenough model [11 13, 16, 17, calling for understanding the emergence of weak $C D$ within the $3+/ 4+$ valence picture.

Another closely related crucial issue is the roles of different microscopic interactions in the observed chargeorbital orders, in particular the relevance of electronelectron $(e-e)$ interactions in comparison with the wellaccepted electron-lattice $(e-l)$ interactions. For example, 
$\Delta n$, the difference in the electron occupation number be tween $\mathrm{Mn}^{3+}$ and $\mathrm{Mn}^{4+}$ states, was shown to be small in a $e$-e only picture [18; this is however insufficient to explai the observed weak $\mathrm{CD}$, since the established $e-l$ interac tions will cause a large $\Delta n[19$. Moreover, despite th common belief that $e-l$ interactions dominate the gener: physics of $e_{g}$ electrons in the manganites [2,20], a recer theoretical study 21] showed that $e-e$ interaction plays a essential and leading role in ordering the $e_{g}$ orbitals in th parent compound. It is thus important to quantify th leading mechanisms in the doped case and uncover the $\mathrm{e}$ fects of the additional charge degree of freedom in genera

In this Letter, we present a general, simple, yet quant: tative picture of doped holes in strongly correlated charge transfer systems, and apply it to resolve the above cor temporary fundamental issues concerning the charge orde in half-doped manganites. Based on recently develope first-principles Wannier states (WSs) analysis [21,22,34 the $\mathrm{LDA}+U$ electronic structure in prototypical Ca-dopt manganites, the doped holes are found to reside primari in the oxygen atoms. They are entirely coherent in sho range [23], forming a Wannier orbital of Mn $e_{g}$ symm try at low-energy scale. This hybrid orbital, together wi the unoccupied Mn $3 d$ orbital, forms the effective "Mn $e_{\text {, }}$ basis in the low-energy theory with conventional $3+/ 4$ valence picture, but simultaneously results in a weak $\mathrm{C}$ owing to the similar degree of mixing with the intrins Mn orbitals, thus reconciling the current conceptual co: tradictions. Moreover, our first-principles derivation of t] low-energy interacting Hamiltonian reveals a surprising essential role of $e$ - $e$ interactions in the observed charge 0 der, contrary to the current lore. Our theoretical methc and the resulting simple picture provide a general fram work to utilize the powerful valence picture even with we; $\mathrm{CD}$, and can be directly applied to a wide range of dopt charge-transfer insulators.

Small CD vs. $3+/ 4+$ valence Picture. - To pr ceed with our WSs analysis, the first-principles electron structure needs to reproduce all the relevant experiment observations, including a band gap of $\sim 1.3 \mathrm{eV}$, CE-tyl magnetic and orbital orders, and weak CD, as well as tv distinct $\mathrm{Mn}$ sites. We find that the criteria are met by t] $\mathrm{LDA}+U(8 \mathrm{eV})[14,24$ band structure of the prototypic half-hoped manganite $\mathrm{La}_{1 / 2} \mathrm{Ca}_{1 / 2} \mathrm{MnO}_{3}$ based on the $\mathrm{r}$ alistic crystal structure [25] supplemented with assum alternating La/Ca order. Hence, a proper analysis of th $\mathrm{LDA}+U$ electronic structure is expected to illustrate t] unified picture of weak $\mathrm{CD}$ with the $\mathrm{Mn}^{3+} / \mathrm{Mn}^{4+}$ assig. ment, which can be easily extended to other cases. In practice, we shall focus on the most relevant low-energy (near the Fermi level $E_{\mathrm{F}}$ ) bands - they are 16 " $e_{g}$ " spinmajority bands (corresponding to 8 "spin-up" Mn atoms in our unit cell) spanning an energy window of $3.2 \mathrm{eV}$, as clearly shown in Fig. 11(a). For short notation, the Mn bridge- and corner-sites in the zigzag ferromagnetic chain are abbreviated to B- and C-sites, respectively.

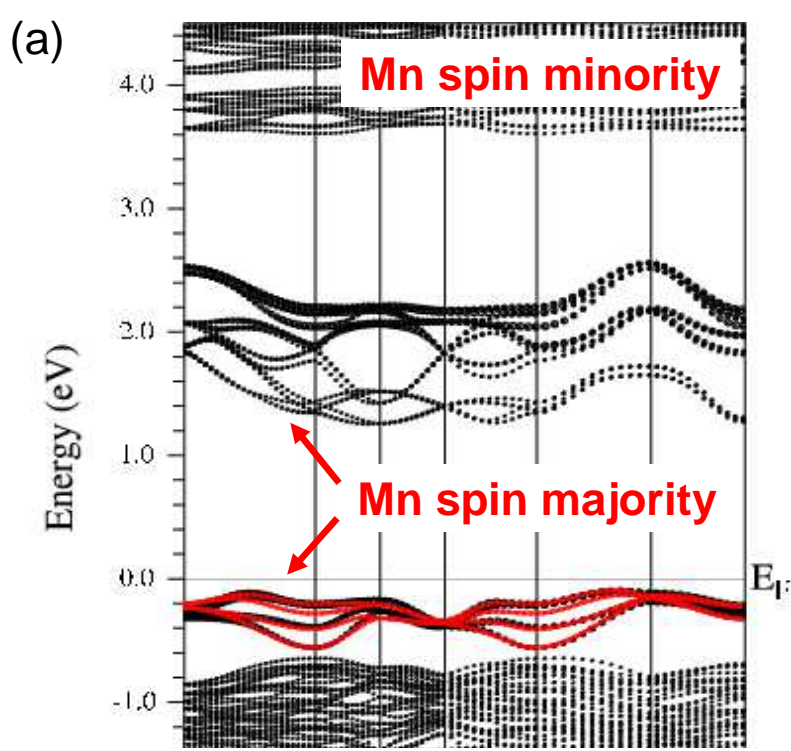

(c)

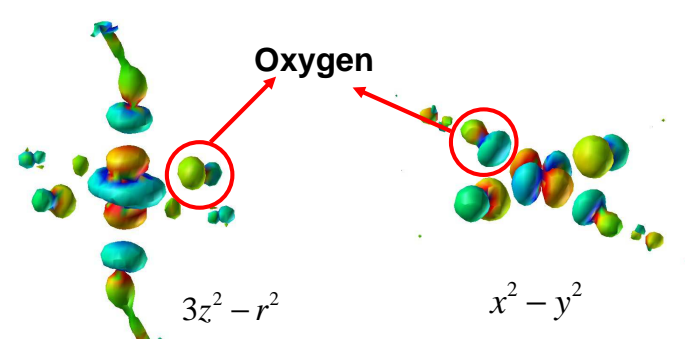

Fig. 1: (Color online) (a) LDA $+U$ Band structures (dots). The (red) lines result from the Wannier states analysis of the four occupied spin-majority Mn 3d-derived bands. (b) An occupied B-site ("Mn ${ }^{3+}$ ") Wannier orbital in a spin-up (up arrow) zigzag chain, showing remarkable delocalization to the neighboring Mn C-sites. (c) Low-energy Mn atomic-like Wannier states containing in their tails the integrated out $\mathrm{O} 2 p$ orbitals.

The simplest yet realistic picture of the $\mathrm{CO}$ can be obtained by constructing occupation-resolved WSs (ORWSs) from the four fully occupied bands, each centered at one B-site as illustrated in Fig. 1(a). This occupied B-site $e_{g}$ Wannier orbital of $3 x^{2}-r^{2}$ or $3 y^{2}-r^{2}$ symmetry (so formal valency is $3+$ ) contains in its tail the integrated out O $2 p$ orbitals with considerable weight, indicative of the 
charge-transfer nature 15. Moreover, this "molecular orbital in the crystal" extends significantly to neighboring C-sites on the same zig-zag chain. Therefore, although by construction the C-site $e_{g}$ ORWSs (not shown) are completely unoccupied (so formal valency is $4+$ ), appreciable charge is still accumulated within the $\mathrm{C}$-site $\mathrm{Mn}$ atomic sphere owing to the large tails of the two occupied ORWSs centered at the two neighboring B-sites. Integrating the charges within the atomic spheres around the B- and C-site $\mathrm{Mn}$ atoms leads to a $\mathrm{CD}$ of mere $0.14 e$, in agreement with experimental $0.1-0.2 e[7-[12$. In this simple picture, one finds a large difference in the occupation numbers of the ORWSs at the $B$ - and $C$ - sites $(\Delta n=1)$, but a small difference in real charge. That is, the convenient $3+/ 4+$ picture is perfectly applicable and it allows weak $\mathrm{CD}$, as long as the itinerant nature of manganites is incorporated via low-energy WSs rather than standard "atomic states."

In comparison, to make connection with the conventional atomic picture and to formulate the spontaneous symmetry breaking with a symmetric starting point (c.f. the next section), we construct from the 16 low-energy bands "atomic-like" WSs (AWSs) of Mn $d_{3 z^{2}-r^{2}}$ and $d_{x^{2}-y^{2}}$ symmetry, as shown in Fig. 1(b). In this picture, both B- and C-site AWSs are partially occupied with $\Delta n=0.6$. Now weak CD results from large hybridization with $\mathrm{O} 2 p$ orbitals, which significantly decreases the charge within the Mn atomic sphere. Obviously, this picture is less convenient for an intuitive and quantitative understanding of weak CD compatible with the $3+/ 4+$ picture than the previous one, as the latter builds the information of the Hamiltonian and the resulting reduced density matrix into the basis. On the other hand, it indeed implies that strong charge-transfer in the system renders it highly inappropriate to associate $C D$ with the difference in the occupation numbers of atomic-like states.

Furthermore, we find that the above conclusions are generic in manganites by also looking into the two end limits of $\mathrm{La}_{1-x} \mathrm{Ca}_{x} \mathrm{MnO}_{3}(x=0,1)$. As shown in Fig. 2, Mn $d$ charge (within the Mn atomic sphere) is found to change only insignificantly upon doping, in agreement with experiments 7,26 . This indicates that doped holes reside primarily in the oxygen atoms, but are entirely coherent in short-range and form additional effective "Mn $e_{g}$ " orbitals in order to gain the most kinetic energy from the $d$ - $p$ hybridization, as shown in Fig. 1(a)-(b), in spirit similar to hole-doped cuprates 23. This justifies the present simplest description of the charge-orbital orders with only the above Mn-centering $e_{g}$ WSs [27].

Leading Mechanisms. - To identify the leading mechanisms of the charge-orbital orders in a rigorous formalism, we proceed to derive a realistic effective lowenergy Hamiltonian, $H^{\text {eff }}$, following our recently developed first-principles WS approach [21,34. As clearly shown in Fig. 1, the low-energy physics concerning charge and orbital orders is mainly the physics of one zig-zag FM

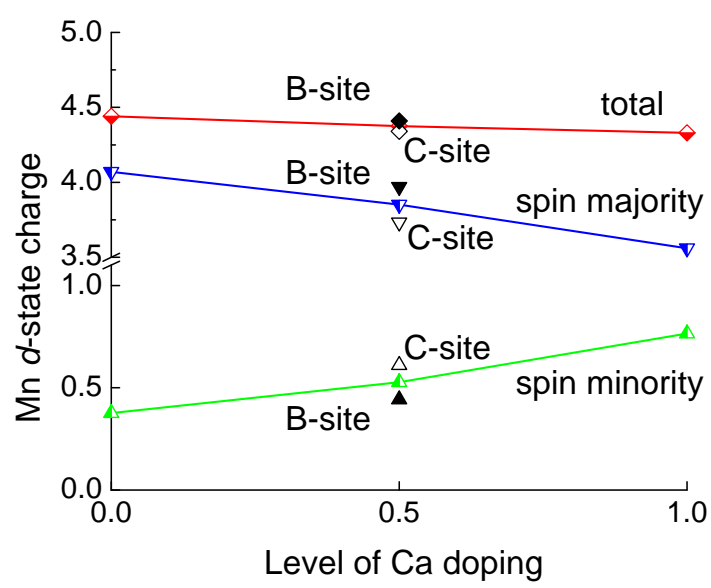

Fig. 2: The calculated $d$ charge within the Mn atomic sphere for $\mathrm{La}_{1-x} \mathrm{Ca}_{x} \mathrm{MnO}_{3}(x=0,1 / 2$, and 1$)$. The results are shown in terms of (i) B-site (filled symbols), C-site (empty symbols), and site-average (half-filled symbols); (ii) spin-majority (up triangles), spin-minority (down triangles), and total charge (diamonds). The lines are guides to eye.

chain, since electron hopping between the antiferromagnetically arranged chains is strongly suppressed by the double-exchange effect [18, 28, 29]. Our unbiased firstprinciples analysis of the 16-band one-particle $\mathrm{LDA}+U$ Hamiltonian in the above AWS representation reveals [18, $28,29,36$

$$
\begin{aligned}
H^{\mathrm{eff}} & =-\sum_{\langle\mathbf{i} \mathbf{j}\rangle \gamma \gamma^{\prime}}\left(t_{\mathbf{i} \mathbf{j}}^{\gamma \gamma^{\prime}} d_{\mathbf{j} \gamma^{\prime}}^{\dagger} d_{\mathbf{i} \gamma}+\text { h.c. }\right)-E_{z} \sum_{\mathbf{i}} T_{\mathbf{i}}^{z} \\
& +U_{\mathrm{eff}} \sum_{\mathbf{i}} n_{\mathbf{i} \uparrow} n_{\mathbf{i} \downarrow}+V \sum_{\langle\mathbf{i} \mathbf{j}\rangle} n_{\mathbf{i}} n_{\mathbf{j}} \\
& -g \sum_{\mathbf{i}}\left(\frac{1}{\sqrt{2}} n_{\mathbf{i}} Q_{1 \mathbf{i}}+T_{\mathbf{i}}^{x} Q_{2 \mathbf{i}}+T_{\mathbf{i}}^{z} Q_{3 \mathbf{i}}\right)
\end{aligned}
$$

in addition to the elastic energy $K\left(\left\{\mathbf{Q}_{\mathbf{i}}\right\}\right)$. Here $d_{\mathbf{i} \gamma}^{\dagger}$ and $d_{\mathbf{i} \gamma}$ are electron creation and annihilation operators at site i with "pseudo-spin" $\gamma$ defined as $|\uparrow\rangle=\left|3 z^{2}-r^{2}\right\rangle$ and $|\downarrow\rangle=\left|y^{2}-x^{2}\right\rangle$ AWSs, corresponding to pseudo-spin operator $T_{\mathbf{i}}^{x}=\left(d_{\mathbf{i} \uparrow}^{\dagger} d_{\mathbf{i} \downarrow}+d_{\mathbf{i} \downarrow}^{\dagger} d_{\mathbf{i} \uparrow}\right) / 2$ and $T_{\mathbf{i}}^{z}=\left(d_{\mathbf{i} \uparrow}^{\dagger} d_{\mathbf{i} \uparrow}-d_{\mathbf{i} \downarrow}^{\dagger} d_{\mathbf{i} \downarrow}\right) / 2$. $n_{\mathbf{i}}=d_{\mathbf{i} \uparrow}^{\dagger} d_{\mathbf{i} \uparrow}+d_{\mathbf{i} \downarrow}^{\dagger} d_{\mathbf{i} \downarrow}$ is the electron occupation number. The in-plane hoppings are basically symmetry related: $t_{\mathbf{i j}}^{\uparrow \uparrow}=t / 4, t_{\mathbf{i j}}^{\downarrow \downarrow}=3 t / 4, t_{\mathbf{i} \mathbf{j}}^{\uparrow \downarrow}=t_{\mathbf{i j}}^{\downarrow \uparrow}= \pm \sqrt{3} t / 4$ where the signs depend on hopping along the $x$ or $y$ direction. $E_{z}$ stands for the oxygen octahedral-tilting induced crystal field. $U_{\text {eff }}$ and $V$ are effective on-site and nearest-neighbor $e-e$ interactions, respectively. $g$ is the $e-l$ coupling constant. $\mathbf{Q}_{\mathbf{i}}=\left(Q_{1 \mathbf{i}}, Q_{2 \mathbf{i}}, Q_{3 \mathbf{i}}\right)$ is the standard octahedral-distortion vector, where $Q_{1 \mathbf{i}}$ is the breathing mode (BM), and $Q_{2 \mathbf{i}}$ and $Q_{3 \mathbf{i}}$ are the Jahn-Teller (JT) modes [18, 19, 28, 29, 36]. In Eq. (1) the electron-lattice couplings have been constrained to be invariant under the transformation of the cubic group [36].

The effective Hamiltonian are determined by matching its self-consistent Hartree-Fock (HF) expression with 
Table 1: Contributions of different terms to the energy gain (in units of $\mathrm{meV}$ per $\mathrm{Mn}$ ) due to the $\mathrm{CO}$ formation in selfconsistent mean-field theory. BM (JT) denotes the contribution from electronic coupling to the breathing (Jahn-Teller) mode. $\mathrm{K}$ denotes that from the elastic energy.

\begin{tabular}{cccccccc}
$\mathbf{Q}_{\mathbf{i}}$ & Total & $U_{\text {eff }}$ & $V$ & $t$ & BM & JT & $K$ \\
\hline 0 & -13 & -11 & -15 & 12 & 0 & 0 & 0 \\
realistic & -127 & -22 & -42 & 71 & -42 & -113 & 24
\end{tabular}

$H^{\mathrm{LDA}+U}$ 21,34 owing to the analytical structure of the $\mathrm{LDA}+U$ approximation [30. An excellent mapping results from $t=0.6 \mathrm{eV}, E_{z}=-0.08 \mathrm{eV}, U_{\text {eff }}=1.65 \mathrm{eV}$, $V=0.44 \mathrm{eV}$, and $g=2.35 \mathrm{eV} / \AA$. These numbers are close to those obtained for undoped $\mathrm{LaMnO}_{3}$ 21] (excluding $V$, which is inert in the undoped case), and indicates that the spin-majority $e_{g}$ electrons in the manganites are still in the intermediate e-e interaction regime with comparable e-l interaction. Note that $U_{\text {eff }}$ should be understood as an effective repulsion of corresponding Mn-centered WS playing the role of "d" states, rather than the "bare" $d-d$ interaction $U=8 \mathrm{eV}$ [21]. Furthermore, the rigidity of the low-energy parameters upon significant $(x=0.5)$ doping verifies the validity of using a single set of parameters for a wide range of doping levels, a common practice that is not a priori justified for low-energy effective Hamiltonians. Clearly, the observed optical gap energy scale of $\sim 2$ $\mathrm{eV}$ originates mainly from $U_{\text {eff }}$ instead of the JT splitting widely assumed in existing theories [5,20].

Now based on the AWSs, CO is measured by $\Delta n=$ $\left\langle n_{\mathbf{i} \in \mathrm{B}}\right\rangle-\left\langle n_{\mathbf{j} \in \mathrm{C}}\right\rangle=0.6$. It deviates from unity because the kinetic energy ensures that the ground state is a hybrid of both B- and C-site AWSs, like in the usual tight-binding modeling based on atomic orbitals. However, since the AWSs considerably extend to neighboring oxygen atoms, the actual CD is much smaller than $\Delta n$.

With the successful derivation of $H^{\text {eff }}$, the microscopic mechanisms of the charge-orbital orders emerge. First of all, note that the kinetic term alone is able to produce the orbital ordered insulating phase [18,29]: Since the intersite interorbital hoppings of the Mn $e_{g}$ electrons along the $x$ and $y$ directions have opposite signs, the occupied bonding state is gapped from the unoccupied nonbonding and antibonding states (by $t$ and $2 t$, respectively) in the enlarged unit cell. As for orbital ordering, the Mn $d_{y^{2}-z^{2}}\left(d_{x^{2}-z^{2}}\right)$ orbital on any B-site bridging two $\mathrm{C}$-sites along the $x(y)$ direction is irrelevant, as the hopping integrals involving it is vanishing. That is, only the $d_{3 x^{2}-r^{2}}\left(d_{3 y^{2}-r^{2}}\right)$ orbital on that B-site is active and the B-sites on the zigzag FM chains have to form an "ordered" pattern of alternating $\left(3 x^{2}-r^{2}\right) /\left(3 y^{2}-r^{2}\right)$ orbitals. However, the kinetic term alone give only $\Delta n=0$. Clearly, $\mathrm{CO}$ is induced by the interactions, $U_{\text {eff }}, V$, or $g$.

To quantify their relative importance for $\mathrm{CO}$, we calculate their individual contributions to the total energy gain with respect to the aforementioned $\Delta n=0$ but orbitalordered insulating state in the self-consistent mean-field theory. The results are listed in Table 1. The first row obtained without lattice distortions provides a measure of the purely electronic mechanisms for CO. Interestingly, although the tendency is weak $(-13 \mathrm{meV})$, e-e interactions all together are sufficient to induce a $C O$, consistent with the results of our first-principles calculations. The second row is obtained for the realistic lattice distortions, which shows a dramatic enhancement of CO by the JT distortions $(-113 \mathrm{meV})$, given that only half of the $\mathrm{Mn}$ atoms are JT active. Together with a $-42 \mathrm{meV}$ gain from the $\mathrm{BM}$ distortion, the $e-l$ interactions overwhelm the cost of the kinetic $(71 \mathrm{meV})$ and elastic $(24 \mathrm{meV})$ energy by -63 $\mathrm{meV}$, further stabilizing the observed CO. Nevertheless, the $-64 \mathrm{meV}$ energy gain from the overall $e$-e interactions accounts for half of the total energy gain, illustrating clearly their importance to the realization of the resulting $\Delta n=0.6$. Indeed, a further analysis reveals that $e-l$ couplings alone $\left(U_{\text {eff }}=V=0\right)$ produce $\Delta n \sim 0.3$, only half of the realistic $\Delta n$, manifesting the necessity of including $e-e$ interactions.

Further insights can be obtained by considering the individual microscopic roles of these interactions acting to the kinetic-only starting point. First, infinitesimal $U_{\text {eff }}$ or $g$ can induce $\mathrm{CO}$, as a result of exploiting the fact that the B- (C-) site has one (two) active $e_{g}$ orbital: (i) $U_{\text {eff }}$ has no effect on the B-sites; therefore, $U_{\text {eff }}$ pushes the $e_{g}$ electrons to the B-sites, in order to lower the Coulomb energy on the C-sites [18. This is opposite to its normal behavior of favoring charge homogeneity in systems such as straight FM chains (realized in the C-type antiferromagnet). (ii) It is favorable to cooperatively induce the $\left(3 x^{2}-r^{2}\right) /\left(3 y^{2}-r^{2}\right)$-type JT distortions on the B-sites and the BM distortions on the $\mathrm{C}$-sites in order to minimize elastic energy [2]. These lattice distortions lower the relative potential energy of the active orbitals in the Bsites, also driving the $e_{g}$ electrons there. Hence, $U_{\text {eff }}$ and $e-l$ interactions work cooperatively in the $\mathrm{CO}$ formation.

Unexpectedly, we find that $V$ alone must be larger than $V_{c} \simeq 0.8 \mathrm{eV}$ to induce CO. This is surprising in comparison to the well-known $V_{c}=0$ for straight FM chains. The existence of $V_{c}$ is in fact a general phenomenon in a "pre-gapped" system. Generally speaking, in a system with a charge gap, $\Delta_{0}$, before CO takes place (e.g. the zigzag chain discussed here), forming $\mathrm{CO}$ always costs non-negligible kinetic energy due to the mixing of states across the gap. As a consequence, unlike the first order energy gain from $U_{\text {eff }}$ and $g$, the second order energy gain from $V$ is insufficient to overcome this cost until $V$ is large enough (of order $\Delta_{0}$ ). In this specific case, $V=0.44 \mathrm{eV}$ is insufficient to induce $\mathrm{CO}$ by itself, but it does contribute significantly to the total energy gain once $\mathrm{CO}$ is triggered by $U_{\text {eff }}$ or $g$, as discussed above.

It is worth mentioning that the contribution from the $E_{z} T_{z}$ term is neglected from Table 1 because of the small coefficient of $E_{z} \simeq 8 \mathrm{meV}$, consistent with the previous 
study for undoped $\mathrm{La}_{3} \mathrm{MnO}_{3}$ 21]. In perovskites, the tilting of the oxygen octahedra could yield the Jahn-Tellerlike distortion of $\mathrm{GdFeO}_{3}$ type, which can be mathematically described by the $E_{z} T_{z}$ or $E_{x} T_{x}$ term. However, in the perovskite manganites these terms are shown here and in Ref. 21 to be negligibly small. In addition, in halfdoped manganites, the pattern of the orbital order is predominantly pined by the zig-zag pathway of the itinerant electrons and thus the effect of tilting is less relevant. On the other hand, the $E_{z} T_{z}$ is very effective to explain the zig-zag orbital ordering of $\left(x^{2}-z^{2}\right) /\left(y^{2}-z^{2}\right)$ pattern in half-doped layered manganites, such as $\mathrm{La}_{0.5} \mathrm{Sr}_{1.5} \mathrm{MnO}_{4}$ Ref. [35], where pseudo-spin-up (the $3 z^{2}-r^{2}$ orbital) is favored by a much stronger $E_{z}$ due to the elongation of the oxygen octahedral along the c-axis. Described via the pseudo-spin angel, $\theta=\arctan \left(T_{x} / T_{z}\right)$ 21, $E_{z}$ significantly reduces $\theta$ from $\pm 120^{\circ}$ [i.e., $\left(3 x^{2}-r^{2}\right) /\left(3 y^{2}-r^{2}\right)$ ] to $\pm 30^{\circ}$ [i.e., $\left(x^{2}-z^{2}\right) /\left(y^{2}-z^{2}\right)$.

The present results would impose stringent constraints on the general understanding of the manganites. For example, Zener polarons were shown to coexist with the $\mathrm{CE}$ phase within a purely electronic modeling of near halfdoped manganites 31]. However, to predict the realistic phase diagram of the manganites, one must take into account the lattice degree of freedom. In fact, when proposing the CE phase, Goodenough considered its advantage of minimizing the strain energy cost. Note that the previous $\mathrm{HF}$ theory indicated a decrease of the total energy by $0.5 \mathrm{eV}$ per unit cell with Zener-polaron-like displacement 15 . The present $\mathrm{LDA}+U$ calculations reveal an increase of the total energy by $1.07 \mathrm{eV}$ per unit cell with the same Zener-polaron-like displacement. This discrepancy is quite understandable from the characteristics of the LDA functional, which favors covalent bond, while the HF approximation tends to over localize the orbital and disfavor bonding. To resolve the competition between the CE phase and the Zener polaron phase, real structural optimization is necessary and will be presented elsewhere. As another example, in the absence of $e-l$ interactions the holes were predicted to localize in the B-site-type region (i.e., the straight segment portion of the zigzag FM chain) in the $\mathrm{C}_{x} \mathrm{E}_{1-x}$ phase of doped E-type manganites [32. However, since the B-sites are susceptible to the JT distortion, they are more likely to favor electron localization instead; future experimental verification is desirable.

Summary. - A general first-principles Wannier function based method and the resulting valence picture of doped holes in strongly correlated charge-transfer systems are presented. Application to the charge order in halfdoped manganites reconciles the current fundamental contradictions between the traditional $3+/ 4+$ valence picture and the recently observed small charge disproportion. In essence, while the doped holes primarily resides in the oxygen atoms, the local orbital are entirely coherent following the symmetry of $\mathrm{Mn} e_{g}$ orbital, giving rise to an effective valence picture with weak CD. Furthermore, our first- principles derivation of realistic low-energy Hamiltonian reveals a surprisingly important role of electron-electron interactions in ordering charges, contrary to current lore. Our theoretical method and the resulting flexible valence picture can be applied to a wide range of doped chargetransfer insulators for realistic investigations and interpretations of the rich properties of the doped holes.

$$
* * *
$$

We thank E. Dagotto for stimulating discussions and V. Ferrari and P. B. Littlewood for clarifying their HartreeFock results [15. The work was supported by U.S. Department of Energy under Contract No. DE-AC02-98CH10886 and DOE-CMSN.

\section{REFERENCES}

[1] Dagotto E., Nanoscale Phase Separation and Colossal Magnetoresistance (Springer-Verlag, Berlin) 2002.

[2] Goodenough J., Phys. Rev., 100 (1955) 564.

[3] Akahoshi D. et al., Phys. Rev. Lett., 90 (2003) 177203; Mathieu R. et al., ibid., 93 (2004) 227202; Takeshita N. et al., Phys. Rev. B, 69 (2004) 180405(R); Alvarez G. et al., ibid., 73 (2006) 224426.

[4] Moreo A. et al., Science, 283 (1999) 2034.

[5] Sen C. et al., Phys. Rev. Lett., 98 (2007) 127202.

[6] Coey M., Nature, 430 (2004) 155.

[7] Tyson T. A. et al., Phys. Rev. B, 60 (1999) 4665.

[8] García J. et al., J. Phys.: Condens. Matter, 13 (2001) 3229.

[9] Daoud-Aladine A. et al., Phys. Rev. Lett., 89 (2002) 097205.

[10] Thomas K. J. et al., Phys. Rev. Lett., 92 (2004) 237204.

[11] Grenier S. et al., Phys. Rev. B, 69 (2004) 134419.

[12] Herrero-Martín J. et al., Phys. Rev. B, 70 (2004) 024408.

[13] Goff R. J. et al., Phys. Rev. B, 70 (2004) 140404.

[14] Anisimov V. I. et al., Phys. Rev. B, 55 (1997) 15494.

[15] Ferrari V. et al., Phys. Rev. Lett., 91 (2003) 277202.

[16] Trokiner A. et al., Phys. Rev. B, 74 (2006) 092403.

[17] Patterson C. H., Phys. Rev. B, 72, 085125 (2005).

[18] van den Brink J. et al., Phys. Rev. Lett., 83 (1999) 5118.

[19] Popović Z. and Satpathy S., Phys. Rev. Lett., 88 (2002) 197201.

[20] Millis A. J., Nature, 392 (1998) 147.

[21] Yin W.-G. et al., Phys. Rev. Lett., 96 (2006) 116405.

[22] Ku W. et al., Phys. Rev. Lett., 89 (2002) 167204.

[23] Zhang F. C. and Rice T. M., Phys. Rev. B, 37 (1988) 3759 .

[24] The WIEN2k [Blaha P. et al., Comput. Phys. Commun., 147 (2002) 71] implementation of the full potential LAPW method is employed.

[25] Radaelli P. G. et al., Phys. Rev. B, 55 (1997) 3015.

[26] Herrero-Martín J. et al., Phys. Rev. B, 72 (2005) 085106.

[27] For explicit inclusion of the oxygen orbitals in a more complete model, see Mostovoy M. V. and Khomskii D. I., Phys. Rev. Lett., 92 (2004) 167201.

[28] Hotta T. et al., Phys. Rev. B, 62 (2000) 9432.

[29] Solovyev I. V. and Terakura K., Phys. Rev. Lett., 83 (1999) 2825. 
[30] Unlike the local interactions, the intersite interactions in $\mathrm{LDA}+U$ is treated via LDA functional, which has dominant Hartree contribution that we used for this mapping.

[31] Efremov D. V. et al., Nature Materials, 3 (2004) 853.

[32] Hotta T. et al., Phys. Rev. Lett., 90 (2003) 247203.

[33] Ch. Jooss et al., PNAS, 104 (2007) 13597.

[34] Yin W.-G. and Ku W., Phys. Rev. B, 79 (2009) 214512.

[35] D. J. Huang et al., Phys. Rev. Lett., 92 (2004) 087202.

[36] P. B. Allen and V. Perebeinos, Phys. Rev. B, 60 (1999) 10747. 